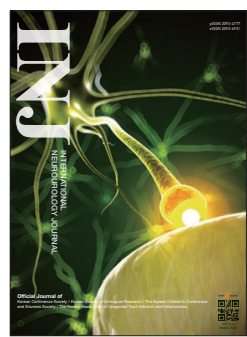

\title{
Is It Possible to Prevent Symptom Recurrence After Transurethral Resection for Hunner Lesion?
}

\author{
Aram Kim ${ }^{1}$, Jung Hyun Shin ${ }^{2}$, Myung-Soo Choo ${ }^{2}$ \\ ${ }^{1}$ Department of Urology, Konkuk University Medical Center, Konkuk University School of Medicine, Seoul, Korea \\ ${ }^{2}$ Department of Urology, Asan Medical Center, University of Ulsan College of Medicine, Seoul, Korea
}

To the Editor:

The debilitating pelvic pain associated with interstitial cystitis/ bladder pain syndrome (IC/BPS) is challenging to treat [1-3]. Transurethral resection and cauterization (TUR-C) is performed to treat patients with Hunner lesions [4]. However, several recent studies have shown that TUR-C alone did not exhibit significant therapeutic effects in patients with IC/BPS [5]; many patients experienced symptom recurrence, although symptom improvement was evident after TUR-C in the short term [6-8]. Bladder instillation therapy refers to the direct introduction of medication into the bladder, and it is a common treatment modality for patients with IC/BPS [8]. Instillation therapy has been shown to be efficacious, but this treatment has never been used to prevent the recurrence of symptoms after TUR-C in patients with Hunner-type IC/BPS. Therefore, we evaluated the preventive effect of additional hyaluronic acid and chondroitin sulfate (HA/CS) instillation therapy after TUR-C.

The study enrolled patients aged 18 years or more who were unresponsive to first-line noninvasive treatments (e.g., oral drugs considered to be standard treatments, such as pentosan polysulfate and amitriptyline). In order to enroll definitive IC/ BPS patients, we used strict criteria and included IC/BPS patients who had a visual analogue scale (VAS) pain score $>4$ and total scores $>13$ on the pelvic pain and urgency/frequency (PUF) questionnaire and $>12$ on the O'Leary-Sant IC symptom index (ICSI)/problem index (ICPI). Hunner lesions were confirmed by cystoscopy. During TUR-C, the Hunner lesions were removed and cauterized to the maximal extent possible, using bi- polar resectoscopy under general anesthesia. In order to assess the preventive effect of intravesical instillation of $\mathrm{HA} / \mathrm{CS}$, we divided the enrolled patients into a group that underwent TUR-C and a group that received additional instillation of HA/CS after TUR-C. These patients received once-weekly intravesical instillations of $40 \mathrm{mg}$ of HA/CS diluted in $50 \mathrm{~mL}$ of saline solution for 4 weeks and once monthly for 2 months.

The primary endpoints were change in pelvic pain from baseline and the recurrence rate of pelvic pain, which was defined as a VAS score $>4$ points at 12 months. The VAS used a $0-10$ scale $(0=$ no pain and $10=$ worst possible pain $)$, and an assessment at 12 months was compared with the baseline. The secondary endpoints were reduction in pain intensity, changes in ICSI/ ICPI scores, and changes in urinary frequency based on a 3-day voiding diary from baseline to 6 months postoperatively. The safety assessment included the registration of all investigatorassessed adverse events. Analysis of covariance was performed to compare the 2 groups, with baseline levels as the covariate and using a modified baseline-observation-carried-forward approach to impute missing data for participants who dropped out due to lack of efficacy or adverse events and a last-observation-carried-forward strategy for patients who dropped out for other reasons. VAS score changes from baseline to 12 months postoperatively (the primary endpoint) were analyzed in the intention to treat population, including all randomized patients, and in the per protocol population, including patients who completed the study without any major protocol violation or receiving any grade $\mathrm{A} / \mathrm{B}$ recommended treatment for IC/BPS,
Corresponding author: Myung-Soo Choo (iD https://orcid.org/0000-0002-3293-4751 Department of Urology, Asan Medical Center, University of Ulsan College of Medicine, 88 Olympic-ro 43 gil, Songpa-gu, Seoul 05505, Korea

E-mail: mschoo@amc.seoul.kr, Tel: +82-2-3010-3735, Fax: +82-2-477-8928

Submitted: March 24, 2018 / Accepted after revision: May 28, 2018 c) (7) $\$$ This is an Open Access article distributed under the terms of the Creative Commons Attribution Non-Commercial License (http://creativecommons.org/licenses/by-nc/4.0/) which permits unrestricted non-commercial use, distribution, and reproduction in any medium, provided the original work is properly cited. 
according to the European Association of Urology criteria.

Fig. 1 shows a flow diagram of the study. A total 30 patients were screened, including 15 for TUR-C alone and 15 for TUR$\mathrm{C}$ with additional HA/CS instillation, with a mean age of 61.4 years (range, 49-72 years). There were 7 patients in whom TUR-C alone was discontinued before the end of the study due to dissatisfaction with treatment $(n=2)$, noncompliance with the protocol $(n=3)$, and loss to follow-up $(n=2)$. Seven other

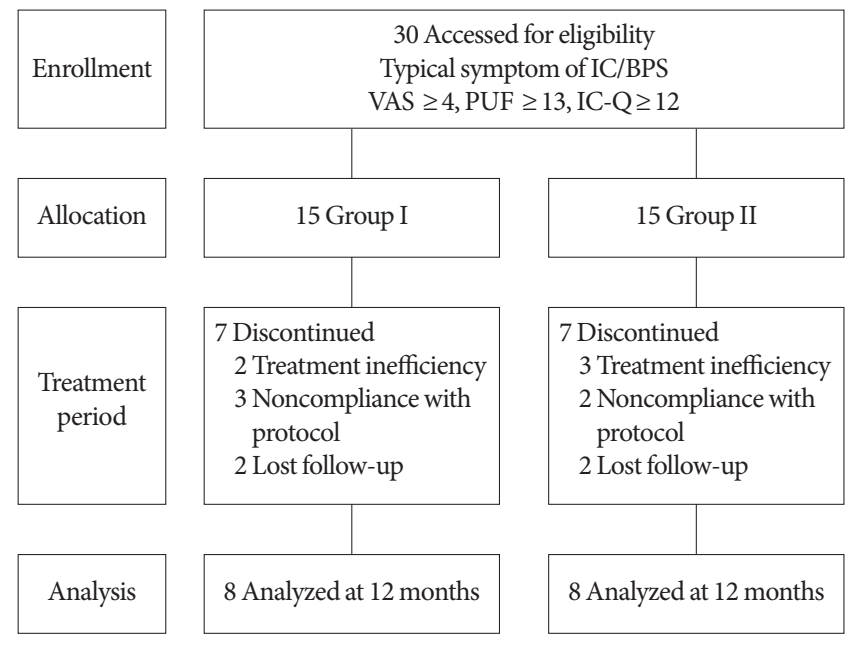

Fig. 1. Diagram of patient selection. IC/BPS, interstitial cystitis/ bladder pain syndrome; TUR-C, transurethral resection and cauterization; group 1, TUR-C only; group 2, TUR-C and instillation; VAS, visual analogue scale; PUF, pelvic pain and urgency/frequency; IC-Q, Interstitial Cystitis-Questionnaire. patients who had TUR-C with additional instillation therapy did not finish the study due to dissatisfaction with treatment $(\mathrm{n}=3)$, noncompliance with the protocol $(\mathrm{n}=2)$, and loss to follow-up $(n=2)$. All patients were randomized and included in the safety analysis. Overall, 16 patients -8 patients $(53.3 \%)$ in the TUR-C alone group and 8 (53.3\%) in the additional instillation group - finished the study. The 2 groups were well represented and well balanced in terms of baseline characteristics. Demographic and baseline clinical characteristics were comparable in the 2 groups at baseline (Table 1). The primary endpoints were change in pelvic pain and the recurrence rate at 12 months after treatment. The patients who underwent TUR-C alone showed a $75 \%$ recurrence rate at 12 months, whereas the patients who received additional HA/CS instillation therapy af-

Table 1. Baseline characteristics

\begin{tabular}{lrrcc}
\hline Characteristic & $\begin{array}{c}\text { Total } \\
(\mathrm{n}=16)\end{array}$ & $\begin{array}{c}\text { Group 1 } \\
(\mathrm{n}=8)\end{array}$ & $\begin{array}{c}\text { Group 2 } \\
(\mathrm{n}=8)\end{array}$ & P-value \\
\hline Age $(\mathrm{yr})$ & $61.4 \pm 7.0$ & $59.8 \pm 7.4$ & $63.0 \pm 6.6$ & 0.391 \\
VAS & $7.3 \pm 1.7$ & $7.6 \pm 1.3$ & $7.0 \pm 2.0$ & 0.482 \\
PUF & $20.6 \pm 6.3$ & $21.8 \pm 5.8$ & $19.3 \pm 6.1$ & 0.461 \\
IC-Q & $25.9 \pm 9.7$ & $27.6 \pm 8.4$ & $24.2 \pm 11.1$ & 0.507 \\
Urinary frequency & $18.5 \pm 2.2$ & $17.8 \pm 2.5$ & $19.1 \pm 1.7$ & 0.275 \\
\hline
\end{tabular}

Values are presented as mean \pm standard deviation.

TUR-C, transurethral resection and cauterization; group 1, TUR-C only; group 2, TUR-C and instillation; VAS, visual analogue scale; PUF, pelvic pain and urgency/frequency; IC-Q, Interstitial Cystitis-Questionnaire.
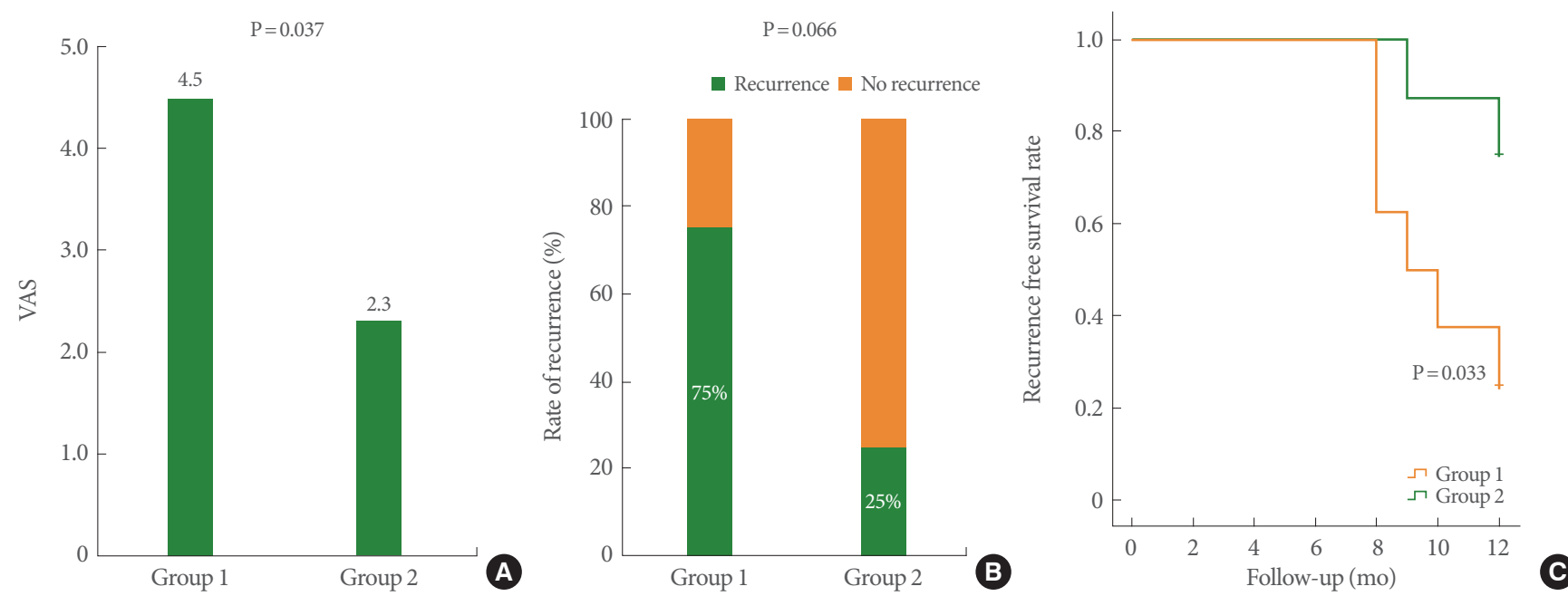

Fig. 2. Comparison of mean visual analogue scale $(V A S)$ score $(P=0.037)(A)$, rate of recurrence $(P=0.066)(B)$, and recurrence-free rate $(\mathrm{P}=0.033)(\mathrm{C})$ between the 2 groups over 12 months of follow-up. TUR-C, transurethral resection and cauterization; group 1 , TUR-C only; group 2, TUR-C and instillation. 

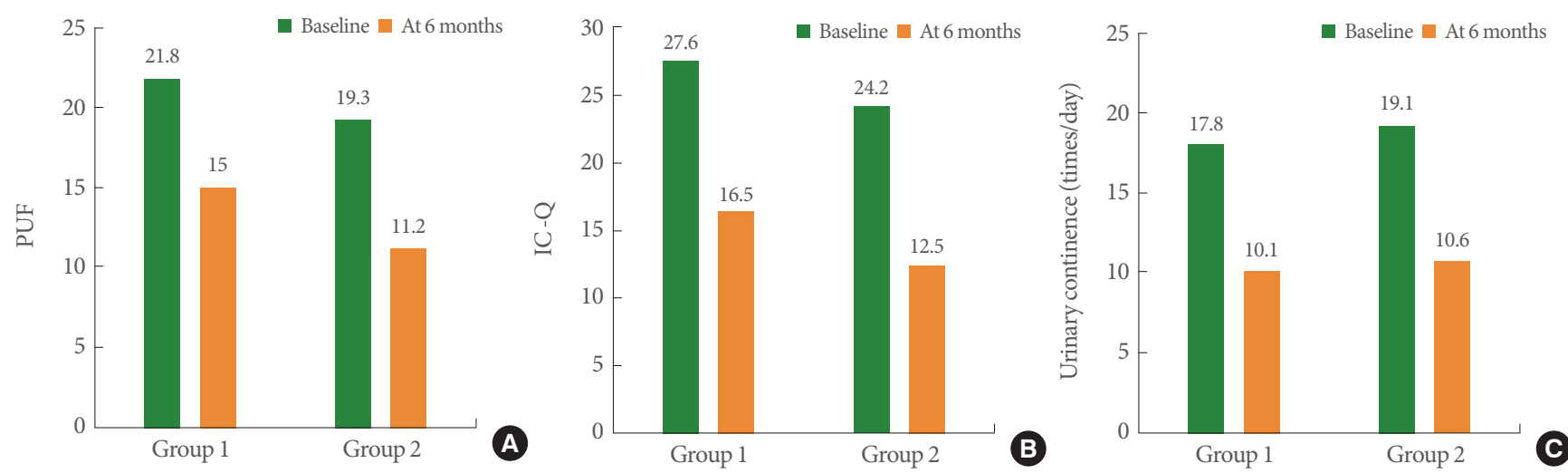

Fig. 3. Comparison of clinical outcomes between the 2 groups at 6 months of follow-up. (A) Pelvic pain and urgency/frequency (PUF), (B) Interstitial Cystitis-Questionnaire (IC-Q), and (C) urinary continence (times/day). TUR-C, transurethral resection and cauterization; group 1, TUR-C only; group 2, TUR-C and instillation.

ter TUR-C showed a recurrence rate of $25 \%$ at 12 months. This difference was significant $(\mathrm{P}<0.001)$ (Fig. 2). A significant reduction in pain intensity was observed at 6 months in both treatment groups versus baseline $(\mathrm{P}<0.001)$; however, the difference in the mean VAS score between the 2 groups did not reach statistical significance at that time. Nonetheless, it reached statistical significance at 12 months ( 3.8 vs. $1.6, \mathrm{P}=0.017$ ). The secondary endpoint was the reduction in PUF scores (-6.8 vs. -8.1 , respectively), total ICSI/ICPI scores ( -11.1 vs. -11.7 , respectively), and urinary frequency ( -7.1 vs. -8.5 , respectively) at 6 months. These reductions were not significantly different between the treatment groups (Fig. 3). No patients showed symptom recurrence, which was defined as a VAS score $>4$ points, at 6 months.

The cause of IC/BPS, which is a chronic and severe inflammatory bladder disease, remains unknown $[9,10]$. HA/CS instillation therapy has shown an encouraging response rate, as highlighted in several recent trials and meta-analyses [11]. Although these treatment strategies have shown good efficacy, a definitive limitation remains; namely, after the alleviation of severe symptoms by treatment, many patients experience symptom recurrence. The results of the present study confirm that both treatments led to reduced pain scores and urinary frequency, as well as improved bladder capacity, in both groups at 6 months after treatment was initiated, and there was no significant difference in efficacy between the 2 groups. No patients showed symptom recurrence at that time. However, there was a significant difference in the rates of symptom recurrence between the 2 groups at 12 months after treatment initiation.

We have reported that TUR-C was efficacious for Hunnertype IC/BPS, although the effect decreased gradually over time
[7]. Generally, instillation treatments are used as a third-line therapy, and are reserved for patients in whom conservative care and oral medication have failed. Unfortunately, several studies have shown that TUR-C only or bladder instillation therapy alone did not exhibit significant long-term therapeutic effects, even though some short-term improvements were evident $[12,13]$. Many treatment tools for IC/BPS have been introduced and evaluated, but no trials have investigated the prevention of symptom recurrence. Therefore, our results might have clinical significance. Severe inflammatory lesions were removed through TUR-C, and defects in the protective barrier of the glycosaminoglycan layer were repaired through HA/CS instillation therapy. Additional instillation therapy after TUR-C might decrease symptom recurrence through the replacement of urothelium throughout the bladder. Some reports have demonstrated that denudation of the entire urothelium of the bladder was found in Hunner-type IC/BPS [14,15]. Additional instillation therapy decreased symptom recurrence, but not Hunner lesions recurrence. We performed cystoscopy at 6 and 12 months after TUR-C in order to evaluate whether Hunner lesions had recurred. Unfortunately, there was no significant difference between the 2 groups regarding the recurrence of Hunner lesions.

The limitations of this study included the small sample size and short follow-up period. In conclusion, additional HA/CS instillation therapy after TUR-C was effective for preventing symptom recurrence in Hunner-type IC/BPS patients.

- Research Ethics: This study was approved by the Institutional Review Board of Asan Medical Center (approval number: 20130135). Clinicaltrial.gov (ID: NCT01813565). Written informed 
consent was obtained from patients.

- Conflict of Interest: No potential conflict of interest relevant to this article was reported.

\section{AUTHOR CONTRIBUTION STATEMENT}

- Full access to all the data in the study and takes responsibility for the integrity of the data and the accuracy of the data analysis: Aram Kim, Jung Hyun Shin, Myung-Soo Choo

- Study concept and design: Aram Kim, Myung-Soo Choo

- Acquisition of data: Aram Kim, Jung Hyun Shin, Myung-Soo Choo

- Analysis and interpretation of data: Aram Kim, Jung Hyun Shin, Myung-Soo Choo

- Drafting of the manuscript: Aram Kim, Jung Hyun Shin, Myung-Soo Choo

- Critical revision of the manuscript for important intellectual content: Aram Kim, Myung-Soo Choo

- Statistical analysis: Aram Kim, Jung Hyun Shin

- Obtained funding: Aram Kim, Myung-Soo Choo

- Administrative, technical, or material support: Aram Kim, Myung-Soo Choo

- Study supervision: Myung-Soo Choo

\section{REFERENCES}

1. McKernan LC, Walsh CG, Reynolds WS, Crofford LJ, Dmochowski RR, Williams DA. Psychosocial co-morbidities in Interstitial Cystitis/Bladder Pain syndrome (IC/BPS): a systematic review. Neurourol Urodyn 2018;37:926-41.

2. Chen IC, Lee MH, Chen WC, Hu TC, Lin HY. Risk factors of lower urinary tract syndrome among ketamine users. Low Urin Tract Symptoms 2017 Jul 13 [Epub]. https://doi.org/10.1111/luts.12178.

3. Kim A, Hoe KO, Shin JH, Choo MS. Evaluation of the incidence and risk factors associated with persistent frequency in interstitial cystitis/bladder pain syndrome and the efficacy of antimuscarinic treatment. Investig Clin Urol 2017;58:353-8.

4. Niimi A, Nomiya A, Yamada Y, Suzuki M, Fujimura T, Fukuhara H, et al. Hydrodistension with or without fulguration of hunner lesions for interstitial cystitis: Long-term outcomes and prognostic predictors. Neurourol Urodyn 2016;35:965-9.
5. Lee SW, Kim WB, Lee KW, Kim JM, Kim YH, Lee B, et al. Transurethral resection alone vs resection combined with therapeutic hydrodistention as treatment for ulcerative interstitial cystitis: initial experience with propensity score matching studies. Urology 2017;99:62-8.

6. Propert KJ, Schaeffer AJ, Brensinger CM, Kusek JW, Nyberg LM, Landis JR. A prospective study of interstitial cystitis: results of longitudinal followup of the interstitial cystitis data base cohort. The Interstitial Cystitis Data Base Study Group. J Urol 2000;163:1434-9.

7. Ryu J, Pak S, Song M, Chun JY, Hong S, Choo MS. Elimination of Hunner's ulcers by fulguration in patients with interstitial cystitis: is it effective and long lasting? Korean J Urol 2013;54:767-71.

8. Colaco MA, Evans RJ. Current recommendations for bladder instillation therapy in the treatment of interstitial cystitis/bladder pain syndrome. Curr Urol Rep 2013;14:442-7.

9. Logadottir Y, Delbro D, Lindholm C, Fall M, Peeker R. Inflammation characteristics in bladder pain syndrome ESSIC type 3C/classic interstitial cystitis. Int J Urol 2014;21 Suppl 1:75-8.

10. Jhang JF, Kuo HC. Pathomechanism of interstitial cystitis/bladder pain syndrome and mapping the heterogeneity of disease. Int Neurourol J 2016;20(Suppl 2):S95-104.

11. Barua JM, Arance I, Angulo JC, Riedl CR. A systematic review and meta-analysis on the efficacy of intravesical therapy for bladder pain syndrome/interstitial cystitis. Int Urogynecol J 2016;27:113747.

12. Hanno PM, Erickson D, Moldwin R, Faraday MM; American Urological Association. Diagnosis and treatment of interstitial cystitis/ bladder pain syndrome: AUA guideline amendment. J Urol 2015; 193:1545-53.

13. Hanno PM, Burks DA, Clemens JQ, Dmochowski RR, Erickson D, Fitzgerald MP, et al. AUA guideline for the diagnosis and treatment of interstitial cystitis/bladder pain syndrome. J Urol 2011;185:216270.

14. Kim A, Han JY, Ryu CM, Yu HY, Lee S, Kim Y, et al. Histopathological characteristics of interstitial cystitis/bladder pain syndrome without Hunner lesion. Histopathology 2017;71:415-24.

15. Peeker R. Re: Hunner-type (classic) interstitial cystitis: a distinct inflammatory disorder characterized by pancystitis, with frequent expansion of clonal B-cells and epithelial denudation. Eur Urol 2016;70:207-8. 